\title{
Numerical Modelling of Rotor-Stator Interaction in Rim Driven Thrusters
}

\author{
Aleksander J. Dubas ${ }^{\mathrm{a}, \mathrm{b}}$, N. W. Bressloff ${ }^{\mathrm{b}}$, S. M. Sharkh ${ }^{\mathrm{b}}$ \\ ${ }^{a}$ Institute of Complex Systems Simulation, University of Southampton, UK \\ ${ }^{b}$ Faculty of Engineering and Environment, University of Southampton, UK
}

\begin{abstract}
An electric rim driven thruster is a relatively new marine propulsion device and the associated fluid dynamics have not been fully investigated. This work develops a robust CFD method and investigates both frozen rotor and unsteady simulations of rotor-stator interaction.

Two solvers from OpenFOAM were used. Steady state simulations were performed using MRFSimpleFoam with a frozen rotor treatment of the interface between static and rotational reference frames. The solver for unsteady simulations was pimpleDyMFoam, utilising a sliding mesh interface to handle the dynamic meshing. Both methods are thoroughly verified and validated against experimental data. The k-omega SST turbulence model is found to be robust down to low advance ratios.

For the rim driven thruster, analytical models are used to estimate friction forces in the rim gap and their contribution to torque losses. The frozen rotor and unsteady treatments of rotor-stator interaction are compared and found to have similar trends in the variation of thrust produced. However, the frozen rotor method does not predict the same variation of instantaneous torque and does not capture the rotor-stator interaction fully. Analysis of the unsteady rotor-stator interaction shows an oscillating flow over the stators and thus inflow to the blades.
\end{abstract}

Keywords:

Frozen Rotor, Unsteady, Numerical, CFD, Thruster, Rim Driven

Email address: ajd205@soton.ac.uk (Aleksander J. Dubas) 
Figure 1: Cross-section diagram of rim driven thruster.

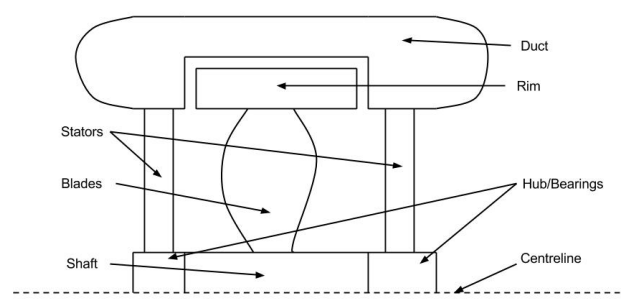

\section{Introduction}

An electric rim driven thruster is a relatively new marine propulsion device which uses a permanent magnet rotor built into a rim around the propeller (Sharkh et al., 2003a). In many ways, rim driven thrusters are hydrodynamically similar to ducted propellers but with a major difference: there is no propeller tip clearance or leakage losses. However, the penalty for this advantage is that there may be leakage around the rim and significant friction losses in the gap between the rim and the duct.

A simplified cross section of the rim driven thruster studied in this paper is shown in Figure 1 with the key parts identified. Starting from the outside, there is a duct which also houses the static parts of the motor. Attached to the duct is a set of hydrodynamic stators which, as the device is bi-directional, serve as both pre-stators and post-stators depending on the direction of thrust. These stators are also a structural component that house and centre the hub and shaft bearings, thus inserted into the stator/hub assembly is the shaft. On the shaft the propeller blades are mounted, and at the blade tips a rim is included which also houses the rotating parts of the motor.

Rim driven thrusters are normally designed to be bi-directional as there is no need for an upstream shaft or gearbox, allowing for a symmetrical geometry and thus equal performance in both operational directions. Lea et al. (2003) investigated commercial rim driven thrusters experimentally and found that they had increased efficiency, flexibility and manoeuvrability compared to conventional propulsors. This was attributed to the torque response of the permanent magnet motors as well as better tip loading and cavitation performance due to the presence of the rim.

A number of different methods have been used previously to model the 
performance and flow field of rim driven thrusters, from boundary element methods to finite volume Reynolds-Averaged Navier Stokes (RANS) methods; the latter being the preferred method in recent literature. Kinnas et al. (2009) conducted a numerical prediction of performance and sheet cavitation of a rim driven tunnel thruster using combined RANS and boundary element methods. The boundary element method was used to calculate thruster performance, which was subsequently fed into a commercial RANS code as a body force to calculate the inflow to the propeller. The inflow profile was then fed iteratively back in to the boundary element code until thrust and torque coefficient had converged. This method predicted thrust very well at high rotational speeds. At low speeds thrust was over-predicted, due to the boundary element method's inability to capture the off-design flow field correctly.

Yakovlev et al. (2011) used a pure RANS code for their modelling, correctly predicting performance over a larger operating range, and applied their code to a design optimisation study of the pitch distribution to produce a $0.4 \%$ increase in efficiency that was subsequently experimentally verified. Additionally, the induced stresses in the blades in various configurations were investigated and it was found that there was a six-fold reduction of peak stress, which is another advantage of a rim drive. This allows designs to be produced with thinner, more hydrodynamically efficient, blades.

Cao et al. (2012) simulated a rim driven thruster using RANS, combined with analytical models to describe the torque production of the TaylorCouette flow in the gap between the rim and duct. This is a large source of losses in rim driven thrusters, contributing to $27 \%$ of the total torque in the work of Cao et al. (2012), although the simulation was simplified through the use of discrete analytical models for the torque. The rim drive thruster studied by Cao et al. differs from the present one by being both hubless and statorless.

In some cases stators have been shown to be beneficial to propulsive efficiency (Celik and Guner, 2007) and it may be possible to exploit the stators in a rim driven thruster. The rim driven thruster investigated in this paper utilises the stators primarily for locating the spindle and bearings, and they may be adapted to improve the hydrodynamic efficiency, but to preserve the bi-directional performance the stators must function as both pre-stators and post-stators.

It is possible to include the rotor-stator interaction either through use of multiple steady-state simulations using a frozen rotor formulation or through 
a more computationally expensive unsteady simulation. The authors are not aware of any previous work on unsteady modelling of rotor-stator interaction in rim driven thrusters. Petit et al. (2009) found that steady-state frozen rotor formulations are not sufficiently accurate for capturing the rotor-stator interaction in a centrifugal pump due to the improper treatment of the impeller wake. However, using a series of frozen rotor simulations at different relative displacements, Li and Wang (2007) modelled the rotor-stator interaction in an axial pump without explicit consideration of the unsteady effects and achieved good results.

The present work builds on previous work by the authors (Dubas et al., 2011), which investigated the steady-state simulation of a rim driven thruster with $70 \mathrm{~mm}$ propeller design and found that the use of steady-state simulations in the presence of stators caused a significant reduction in accuracy due to the poor modelling of the rotor-stator interaction. A similar modelling method to that of Cao et al. (2012) is used in the present work, excluding the rim gap from the CFD and instead modelling its effects analytically, but this geometry also requires simulation of the rotor-stator interaction. To investigate improving the capture of rotor-stator interaction, this work uses two methods, one steady-state and one time-varying, and compares the effect of rotor-stator interaction between the two methods.

\section{Methods}

\subsection{Meshing}

The rim driven thruster used in this study is a $100 \mathrm{~mm}$ diameter bidirectional thruster, similar to the one pictured in Figure 2. This device was selected due to the availability of experimental thrust and torque data. Import of the geometry into the meshing program was through the .stl file format from the SolidWorks geometry originally provided.

The mesh generation was subsequently performed using the blockMesh and snappyHexMesh applications within OpenFOAM. A base hexahedral mesh with an edge length of one half of the propeller diameter was generated using blockMesh. The computational domain extents were also defined in the base mesh (see Figure 3), with the final domain extending five propeller diameters upstream of the device, six diameters in the radial direction and ten diameters downstream of the device; these dimensions were determined based on the verification procedure discussed in Section 2.2. The boundary

conditions were set to a constant velocity inlet, with a symmetry plane in the 
Figure 2: Integrated Thruster by TSL Technology Ltd.

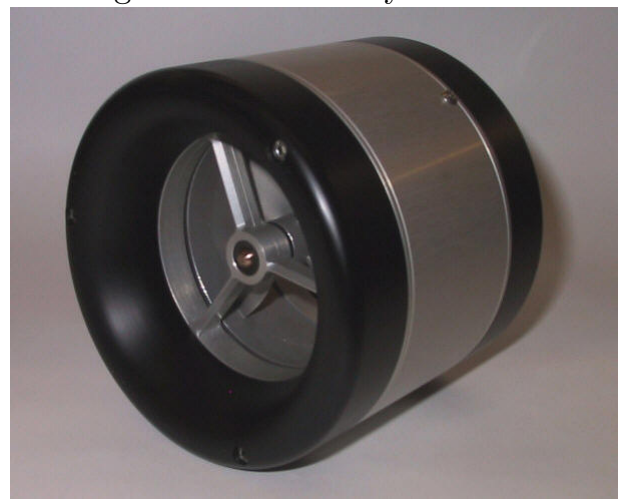

Figure 3: Rim driven thruster meshed domain showing distances to boundaries where $D_{P}$ is the propeller diameter.

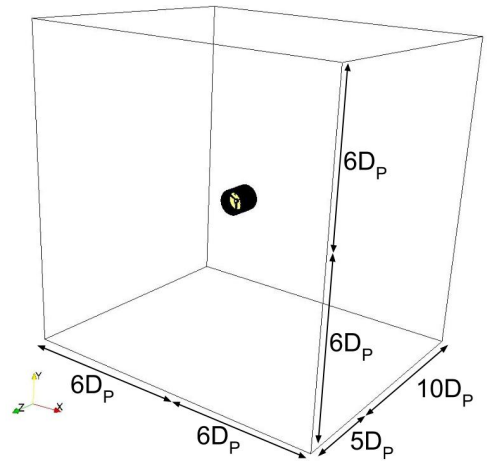

Figure 4: Rim driven thruster surface mesh with rotating surfaces in blue, static surfaces in black.

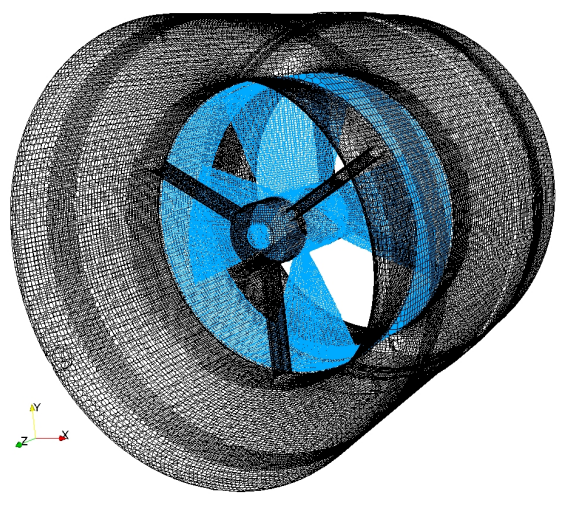


radial directions, that is the positive and negative $x$ and $y$ Cartesian directions, and a constant pressure outlet. On the propeller surface, the mesh edge length was set to $1 / 256^{\text {th }}$ of the propeller diameter, with no wall functions used as the average $y_{1}^{+}$was four and for visual reference the meshed surface is shown in Figure 4. For meshing of the open water propeller, the rotating reference frame region had a radius of 1.14 times the propeller radius, extending past the propeller tips. It has been shown that the size of this region needs to be sufficiently large and is critical to accuracy when using Multiple Reference Frame (MRF) methods for open water propellers (Kaufmann and Bertram, 2011). In the case of the rim driven thruster, the radius of the rotating region extends outside of the fluid domain, in the annulus, which is not simulated in this work due to the increased computational cost of doing so.

As the unsteady simulations were using an Unsteady RANS (URANS) turbulence model, there were no differing requirements on the meshing compared with the steady simulations. However, some method for the movement of the mesh was required, which was chosen to be the Arbitrary Mesh Interface (AMI) implemented in OpenFOAM version 2.1.0 based on the algorithm by Farrell and Maddison (2011). This method involves two topologically disconnected sub-domains, often referred to as a sliding mesh approach, with the flux of variables across the adjoining boundaries distributed with a conservative weighting to connect the sub-domains on each time step at the necessary orientation.

\subsection{Steady State Simulation Method}

For verification and validation of the simulation, a Wageningen B4-70 open water propeller (Kuiper, 1992) of the same dimensions was chosen as the geometry as it has well established and published experimental performance data. To make sure that the solution was not mesh dependent, a mesh verification study was conducted to establish a necessary but sufficient resolution (i.e. edge length) and domain size. For the domain dimensions to be sufficiently large prior to the verification study, a domain extending six propeller diameters both upstream and radially from the device, and 12 propeller diameters downstream, was used. First a surface resolution was established by decreasing the surface edge length (and thus increasing the number of cells) until the result did not change with increasing mesh resolution. The results are shown in Figure 5 and the chosen resolution corresponded to a 
Figure 5: Variation in propeller thrust with number of cells.

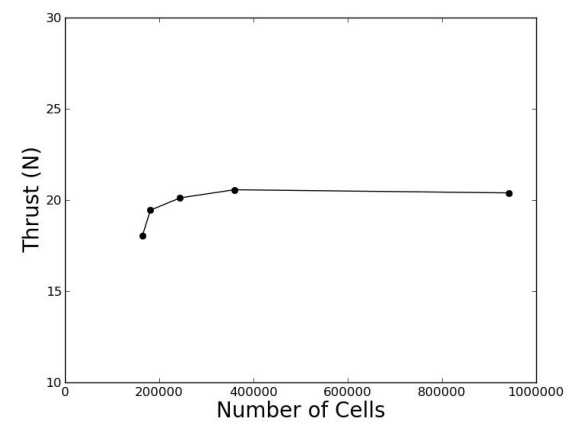

Figure 6: Variation in propeller thrust with distance to inlet boundary.

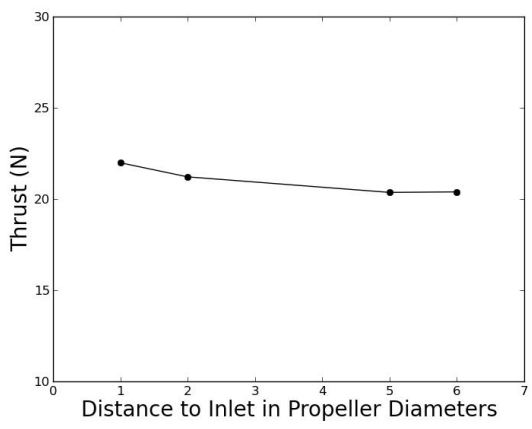

refinement of seven times the base mesh, or $1 / 256^{\text {th }}$ of the propeller diameter.

Similarly, to calculate the domain sizing, the distances to the boundary were varied and the smallest distance at which no further change is seen was selected. While one boundary distance was varied, the others were fixed at the baseline values of six propeller diameters upstream and radially, and 12 downstream. Figure 6 shows the study for the inlet distance, with a distance of five propeller diameters being sufficient in this case. The distance to the boundaries in the radial direction was found to have a profound impact on the accuracy of the result (Figure 7), though a distance of six propeller diameters is sufficient to produce a good result. In the mesh verification study, the distance to the outlet boundary did not seem to have much effect on the result, as seen in Figure 8. However, a value of double the distance to the inlet boundary, that is ten propeller diameters, was selected to allow 
Figure 7: Variation in propeller thrust with distance to radial boundaries.

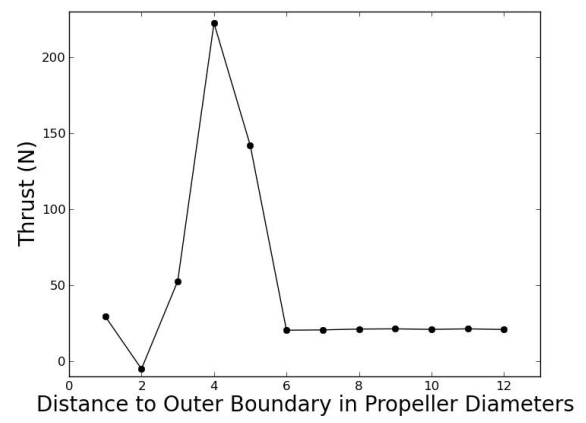

Figure 8: Variation in propeller thrust with distance to outlet boundary.

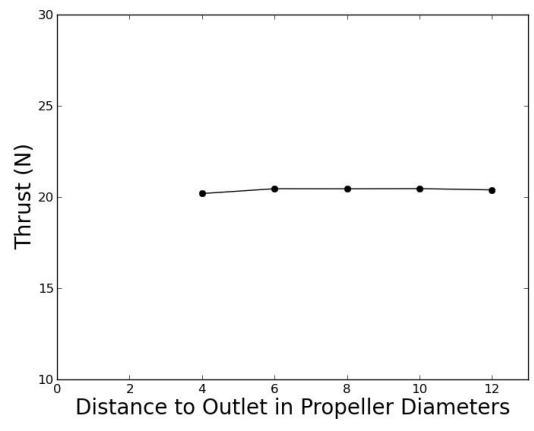

the flow to develop downstream of the propeller.

Once the mesh verification procedure was completed, the solution process was performed on the Wageningen B4-70 propeller and compared against the experimental data published by Kuiper (1992). Initially, the Re-Normalisation Group (RNG) $k-\epsilon$ turbulence model was employed as it is good for rotational flows. However, the RNG $k-\epsilon$ simulations did not converge at low advance ratios, thus the turbulence model was changed to the $k-\omega$ Shear Stress Transport (SST) model and the robustness was improved.

As can be seen in Figure 9, there is a very good agreement between the experimental and computational results. At low advance ratios, there is a divergence between the CFD and experiment, most notably at the bollard pull condition (Advance ratio, $J=0$ ). However this is due to the CFD producing results for a sustained bollard pull, where the induced flow is allowed to establish itself and effectively increase the advance ratio. whereas 
Figure 9: Comparison against experimental data for Wageningen B4-70 propeller.

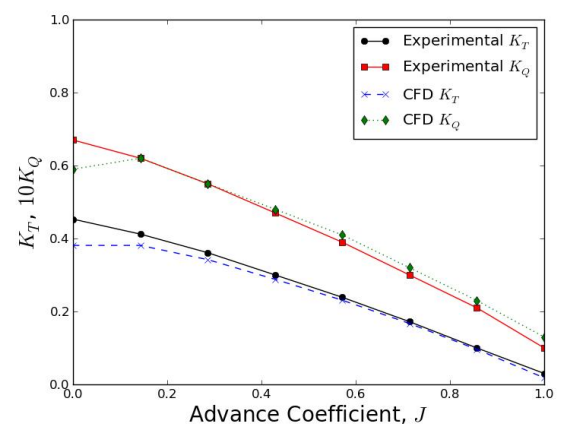

the published experimental data is quoting the instantaneous bollard pull, where both the actual and effective advance ratios are equal to zero, thus reporting a higher thrust than that calculated by the CFD. In reality, the instantaneous bollard pull condition is a transient, and the sustained value is more reflective of real world performance.

In applying the CFD to the rim driven thruster, a further consideration needs to be made for the flow in the annulus between the motor rotor and stator. The flow in this region can be approximated to be a Taylor-Couette flow, which can take on many different states depending on the Reynolds and Taylor numbers of the regime. It is possible to consider the flow in this region through explicit simulation in the computational fluid dynamics code, however to sufficiently resolve this region both spatially and temporally is prohibitively expensive. For the purposes of this work, the prediction of the device performance is the primary aim and the flow regime in the rim gap region is only of secondary interest. It is therefore sufficient to simply consider the torque losses that are generated in the annulus region. This is achieved by using the empirical relationships derived by Bilgen and Boulos (1973) (listed in Equations (1) and (2)) from both their own and previously published experimental work on Taylor-Couette flows.

$$
\begin{gathered}
C_{f}=0.515 \frac{\left(\frac{\delta}{r_{1}}\right)^{0.3}}{R e_{\delta}^{0.5}} \quad\left(500<R e_{\delta}<10^{4}\right) \\
C_{f}=0.0325 \frac{\left(\frac{\delta}{r_{1}}\right)^{0.3}}{R e_{\delta}^{0.2}} \quad\left(10^{4}<R e_{\delta}\right)
\end{gathered}
$$

where $C_{f}$ is the friction coefficient, $r_{1}$ is the inner radius of the gap, $\delta$ is the 
Figure 10: Illustration of rotor angle variation in frozen rotor method.

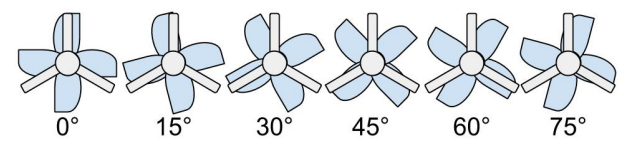

gap width and $R e_{\delta}$ is the Reynolds number based on the gap width, $\delta$.

More recent experiments by Batten (2002) also concur with the Bilgen and Boulos model. However, this model does not account for the effect of the axial gap between the front of the rotor rim and the device housing or the similar gap at the rear of the rotor. A study of the axial gap flow and its effect on torque was published by Daily and Nece (1960), which analysed four different regimes and came up with four formulae, of which the relevant regime to the present case is shown in Equation (3).

$$
C_{f}=\frac{0.08}{\left(\frac{s}{r_{1}}\right)^{0.167} R e_{r}^{0.25}}
$$

where $s$ is the axial spacing, $r_{1}$ is the inner radius (in this case, also the propeller radius) and $R e_{r}$ is the Reynolds number based on the radius, $r_{1}$.

What has not yet been considered is the effect of the axial pressure gradient, which has been shown in some cases to reduce the torque requirement (Manna and Vacca, 2009), although the Taylor numbers in the present study are much higher than covered by Manna and Vacca thus the observed torque reduction is unlikely to manifest here. A formula for the effect of axial pressure gradient on friction torque was developed by Yamada (1962) although it is based on the axial Reynolds number which is not known in the present work and consequently cannot be used to improve the torque estimation.

\subsection{Frozen Rotor Method}

The rotor-stator interaction in the steady state calculations is simulated by what is known as the frozen rotor method. This is a simplification of what is inherently unsteady flow by solving both the rotating and static reference frames in the steady state without any change to their relative position, hence the term frozen rotor.

By solving for multiple rotor angles, as illustrated by Figure 10, the frozen rotor method can be used to produce quasi-unsteady results. This comes at a lower computational cost than full unsteady simulation and may be 
Figure 11: Variation in propeller thrust with distance to radial boundaries for unsteady simulations of Wageningen B4-70 propeller.

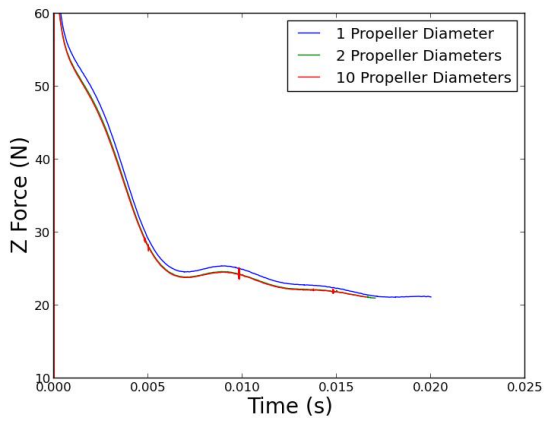

conducted at as many or few rotor angles as desired. The mean performance can then be calculated by averaging over the period of one rotation.

\subsection{Unsteady Simulation Method}

The unsteady solution was conducted using the pimpleDyMFoam solver, which utilises a hybrid PISO (Pressure Implicit Splitting of Operators) and SIMPLE (Semi-Implicit Method for Pressure Linked Equations) algorithm for fast but stable time stepping. This OpenFOAM solver is a dynamic meshing solver, where the mesh motion is through a sliding mesh interface between the rotating and stationary domains. Both first order forward differencing and second order backward differencing time discretisations were tested, and the second order accurate backward differencing method was found to be stable enough to be used throughout the start-up phase from the initial state of a quiescent medium. Consequently it was chosen for its higher order accuracy. The time step that was found to produce a stable solution in a short a time as possible was 0.5 degrees of rotation per time step, which for a rotational rate of 3000 revolutions per minute is approximately $2.5 \times 10^{-5}$ seconds. A total of 0.06 seconds, that is three complete revolutions of the propeller, were required to reach a steady-state from the initial condition. After this time, the transient start-up was finished and only fluctuations about the steady state were recorded.

As for the steady-state simulation, the unsteady simulations also underwent a mesh verification process. It was found that the minimum domain size was significantly smaller than for the frozen rotor method. Figure 11 shows that the required distance to the radial walls was as little as two propeller 
diameters, it was also found that the inlet and outlet boundaries required one and two propeller diameters of distance respectively. However, when the outlet boundary was too close, the solution diverged, thus for robustness a distance of four propeller diameters was chosen here.

For the purposes of validation, the efficiency at the design condition of 3000 revolutions per minute and 1.5 metres per second advance speed was compared, and found to be within $5 \%$ of the experimental value. It should also be noted that there was no time variance of the open water propeller, which was to be expected as there is no source of inherent unsteadiness.

\subsection{Experimental Results}

While the experimental results are not the focus of this paper, it is useful to document the method used by TSL Technology Ltd to obtain them. The experiments were conducted according to the method used by Sharkh et al. (2003b) where the thruster was mounted to a towing tank carriage via a load cell. The forces were measured with the carriage moving at a number of speeds and with different levels of input power to cover a range of advance ratios.

The thrust was calculated by subtracting the drag from the mounting equipment, measured in a separate test, from the measured force to get the equivalent force on the thruster alone. The torque measurements were extrapolated from input power by subtracting estimates of the electromechanical losses to leave only the power absorbed hydrodynamically and then divided by rotation rate to yield torque.

\section{Results \& Discussion}

The rim driven thruster geometry was solved using MRFSimpleFoam. To compare against experimental data, a number of advance ratios were simulated by varying the rotation rate of the rotor at a fixed advance speed of $1.5 \mathrm{~m} / \mathrm{s}$. The advance ratio was changed in this manner to keep the Reynolds number effects the same as the experimental data. The raw results for these simulations can be seen in Figures 12, 13 and 14 showing the thrust

coefficient, torque coefficient and efficiency, respectively. These are defined in Equations (4) to (7) below:

$$
J=\frac{V_{a}}{n D}
$$


Figure 12: Rim driven thruster thrust predicted by the frozen rotor method.

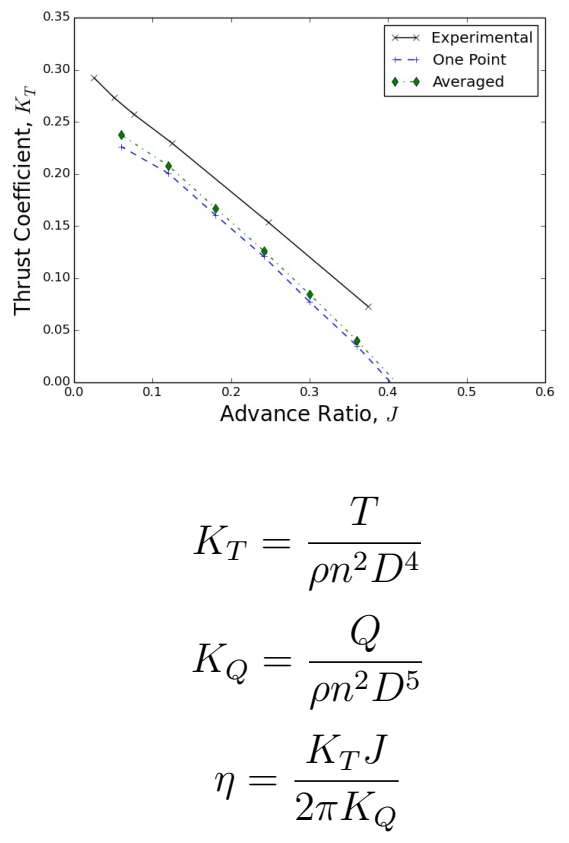

where $J$ is the advance ratio, $V_{a}$ is the advance speed, $n$ is the number of revolutions per second, $D$ is the propeller diameter, $r h o$ is the fluid density, $T$ is the total thrust produced by the device, $Q$ is the total torque absorbed by the rotor including the annulus losses and friction on the shaft and rim, $K_{T}$ is the thrust coefficient, $K_{Q}$ is the torque coefficient and $\eta$ is the efficiency.

The results in Figures 12, 13 and 14 include a naive implementation of the frozen-rotor method where only one point through the rotation is considered, in this case corresponding to zero degrees of rotation, that is one blade aligned with one of the upstream stators. This highlights the need to consider the entire rotation of the device when using the frozen-rotor method to model rotor-stator interaction. As these simulation results do not include the modification of the rim-gap flow, it is expected that an under-prediction in torque should occur as seen in Figure 13. What is interesting, is the underprediction of thrust in Figure 12, which should equal the experimental thrust values without any further modification.

Augmentation of the results with a combination of the analytical rim friction models yields the results in Figures 15 and 16. While this brings the predicted torque to within $10 \%$ of the experimental values in Figure 15, the under-prediction of thrust from Figure 12 cancels out the error in torque 
Figure 13: Rim driven thruster torque predicted by the frozen rotor method.

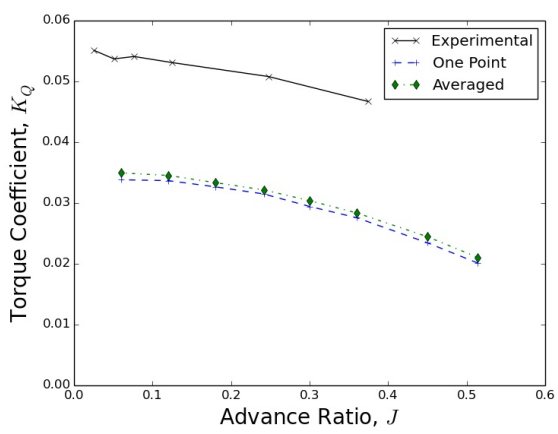

Figure 14: Rim driven thruster efficiency predicted by the frozen rotor method.

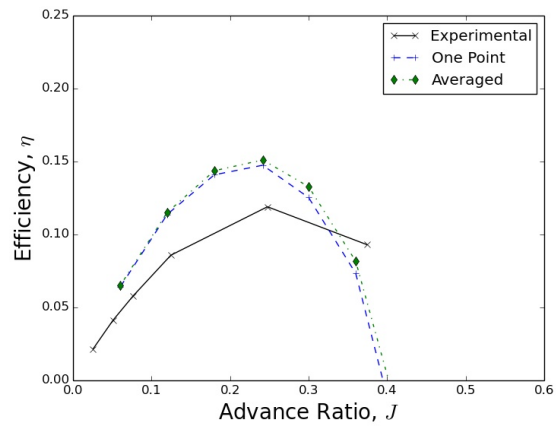

Figure 15: Rim driven thruster torque predicted by the frozen rotor method with and without analytical rim torque correction.

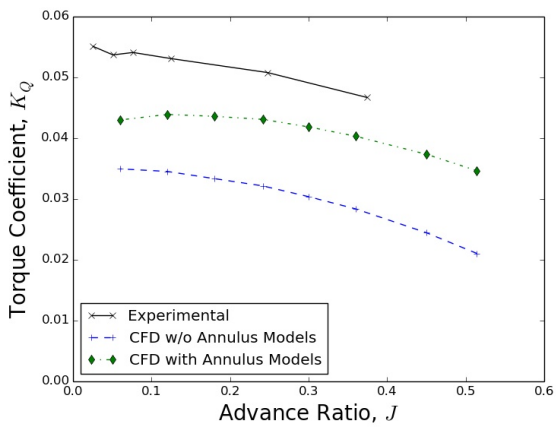


prediction to reduce the difference between numerical and experimental efficiency in Figure 16.

A potential source of the difference between the experimental and numerical results is the testing procedure for the experimental method as detailed in Section 2.5. This involves a support structure which was towed and the thrust measured both with and without the thruster; the latter being deducted from the former to try and isolate the performance of the thruster in solitude. However, the pressure field induced by the propeller may reduce the drag experienced by the support when thrust is produced, thus leading to the experiment predicting a higher thrust than the CFD.

Torque is under-predicted once the analytical models are applied to the CFD results, as shown in Figure 15. As the rotor rim is made as short (in the axial direction) as possible to reduce its area and consequently the friction losses, this leads to a relatively low aspect ratio in the radial gap which may explain the error in prediction of torque as the Bilgen and Boulos model does not cater for end effects which become significant at low aspect ratios. Similarly, to maintain compactness of the device, the rim is made as thin (in the radial direction) as possible, thus leading to a low aspect ratio in the axial gap, increasing the significance of the end effects and also the significance of the interaction with the radial gap flow. The end effects are critical to the resulting flow in this geometry and the interactivity between the axial and radial gap flows should not be excluded. In addition to this, the present analytical models do not account for the effects of an axial pressure gradient, which has been shown by Yamada (1962) to increase the torque requirement in some cases, and that may explain some of the torque under-prediction here.

It may also be the case that the experimental data are not completely accurate as the torque is calculated from input electrical power and estimates of electromechanical losses as described in Section 2.5. While the input electrical power is accurately measurable, the accuracy of the torque value given by the experiments depends greatly upon the accuracy of the estimates of electromechanical losses.

The overall effect of the augmentation of the analytical models on the efficiency is shown in Figure 16. In the region up to an advance ratio of 0.3 the modified results are a closer prediction to the experimental results, due to the combination of under-predicted thrust and torque, but the correlation diminishes as the thrust predicted by CFD (and consequently estimated efficiency) approaches zero. The models also make the shape and response of the 
results more like the experimental results and do not feature the substantial drop off in efficiency above an advance ratio of 0.3 that is present in the raw CFD results.

Solution of the rim driven thruster was also made using the frozen rotor simulation with varying relative angle of the blades to the stators. In addition, an unsteady simulation of the same geometry using pimpleDyMFoam was run such that the unsteadiness caused by the rotor-stator interaction, and the capture thereof by the frozen rotor method, could be investigated.

The variation in thrust and torque over multiple periods of the unsteady simulation and one quarter rotation simulated by the frozen rotor method, as the device is rotationally symmetric every 90 degrees as there are four blades, are overlaid in Figures 17 and 18. It would seem from Figure 17 that the capture of the unsteady variation by the frozen rotor method is within the bounds of periodic variation. However, examining the torque in Figure 18 tells a different story, with a significant difference between the unsteady and frozen rotor simulations.

Further analysis of the unsteady flow past the stators reveals interesting behaviour in the flow. In Figure 19 the streamlines past a stator with no blade behind it can be seen and show a slight deflection of the flow to the right of the figure, which is the direction of rotation. Shortly after, when the next blade has moved in to position behind the stator, Figure 20 shows a deflection of the flow past the stator in the opposite direction. There are two consequences to this effect, the first is the stators are experience an oscillating flow over themselves, which occurs at a frequency of $N$ times per rotation where $N$ is the number of blades. As there are four blades at a rotation rate of 3000 revolutions per minute in this case, the frequency of oscillation is $200 \mathrm{~Hz}$, that is one period as shown in Figures 17 and 18. Secondly, the oscillating flow past the stators will cause an oscillating inflow to the blades which, if one considers the velocity triangle at the blade section, will cause a variation in the effective angle of attack. This oscillation is compounded with the effect of the axial velocity deficit behind the stators causing the multiple peaks in the variation in thrust and torque in Figures 17 and 18 respectively. This has implications on the design of the blades as, on average, the blades may be operating at a different angle of attack than expected and may suffer higher instantaneous peak loads when at the maximum angle of attack in the cycle. 
Figure 16: Rim driven thruster efficiency predicted by the frozen rotor method with and without analytical rim torque correction.

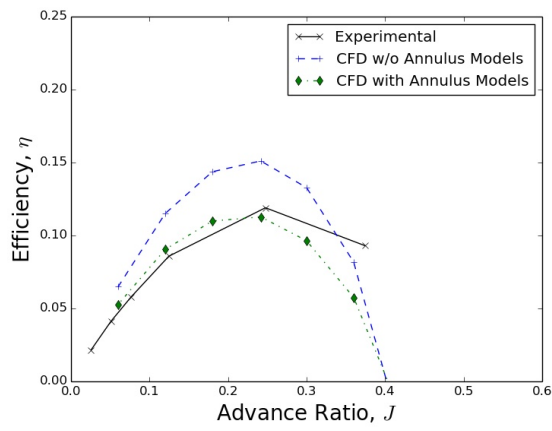

Figure 17: Variation in thrust with time over one quarter revolution, normalised against the time-averaged value of thrust.

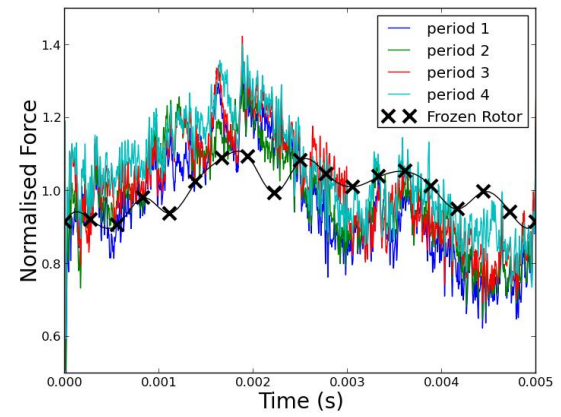

Figure 18: Variation in torque with time over one quarter revolution, normalised against the time-averaged value of torque.

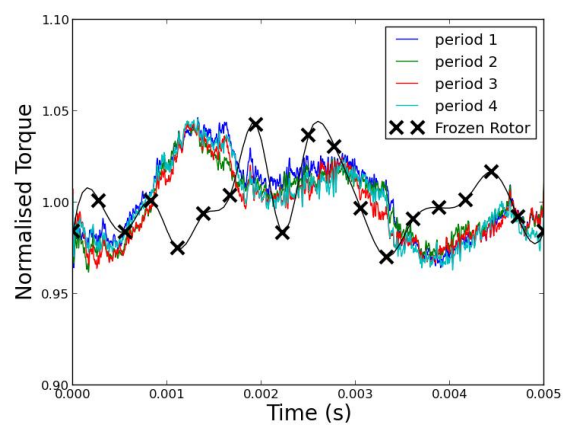


Figure 19: Streamlines past an upstream stator with rotor angle of 50 degrees.

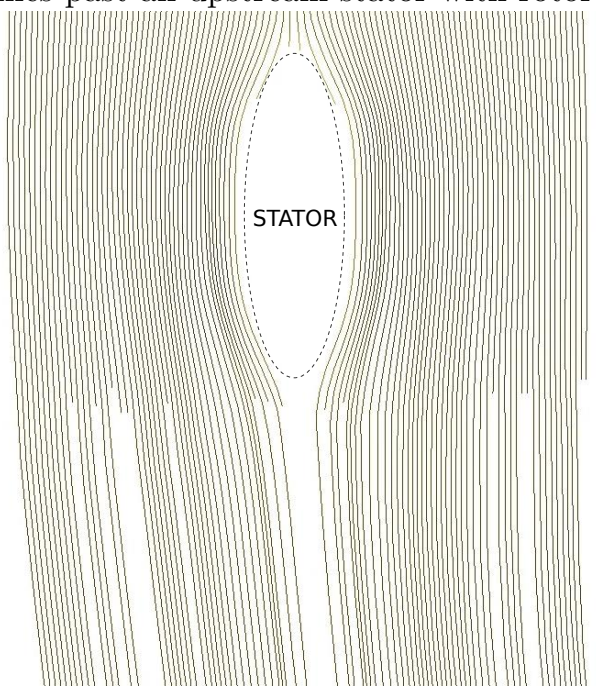

Figure 20: Streamlines past an upstream stator with rotor angle of 90 degrees.

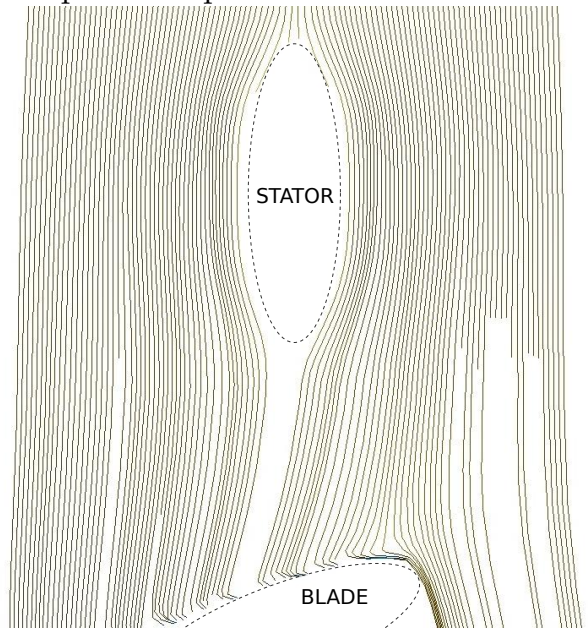




\section{Conclusions}

Two methods for the numerical solution for the performance of rim driven thrusters and marine propulsors in general have been presented. They have subsequently been thoroughly verified and validated, with a recommended domain size and mesh resolution being prescribed. Two turbulence models were compared, the RNG $k-\epsilon$ and $k-\omega$ SST models, and the $k-\omega$ SST model was found to be more robust for solving low advance ratios.

Analytical models for the axial and radial rim gaps have been investigated in conjunction with computational fluid dynamics that solves the flow field external to the annulus. A combination of the Daily \& Nece and Bilgen \& Boulos models for torque contributions are found to under-predict the torque, which may be attributed to low aspect ratio in the former and axial pressure gradient effects in the latter. It is possible that there are also torque effects from the interactivity between the two gap flows. Accurately estimating the torque in the axial and radial rim gaps is desirable not only in rim driven thrusters but a plethora of rotating machinery; therefore further research in this area would be of considerable value to a number of applications.

The capture of the unsteady rotor-stator interaction by the frozen-rotor method has been investigated and while there is some good qualitative matching, it is lacking in accuracy, especially for torque prediction. In conclusion the frozen rotor method is not suitable for capturing the full unsteadiness involved in rotor-stator interaction, but could make a good approximation if the result is required for a relatively cheap computational cost, as in an iterative design optimisation study, for example. However, if time accurate results

are necessary, the authors recommend the use of a sliding mesh approach as employed in the unsteady simulations in this work. The unsteady simulations of the rotor-stator interaction show an oscillating effect on inflow angle to the stators, and consequently the blades, which has design implications on pitch distribution selection.

\section{Acknowledgements}

This work was supported by an EPSRC Doctoral Training Centre grant (EP/G03690X/1). The rim driven thruster geometry and some assistance with generating the open water propeller geometry were provided by TSL Technology Ltd. The authors acknowledge the use of the IRIDIS High Performance Computing Facility, and associated support services at the University of Southampton, in the completion of this work. 


\section{References}

Batten, W. M. J., August 2002. Numerical predictions and experimental analysis of small clearance ratio Taylor-Couette flows. Ph.D. thesis, University of Southampton, School of Engineering Sciences.

Bilgen, E., Boulos, R., 1973. Functional dependence of torque coefficient of coaxial cylinders on gap width and reynolds numbers. Transactions of ASME, Journal of Fluids Engineering 95 (1), 122-126.

Cao, Q.-M., Hong, F.-W., Tang, D.-H., Hu, F.-L., Lu, L.-Z., 2012. Prediction of loading distribution and hydrodynamic measurements for propellers blades in a rim driven thruster. Journal of Hydrodynamics 24, 50-57.

Celik, F., Guner, M., 2007. Energy saving device of stator for marine propellers. Ocean Engineering 34, 850-855.

Daily, J. W., Nece, R. E., 1960. Chamber dimension effects on induced flow and frictional resistance of enclosed rotating disks. Journal of Fluids Engineering 82, 217-230.

Dubas, A. J., Bressloff, N. W., Fangohr, H., Sharkh, S. M., 2011. Computational fluid dynamics simulation of a rim driven thruster. In: Proceedings of the Open Source CFD International Conference, Paris.

Farrell, P. E., Maddison, J. R., 2011. Conservative interpolation between volume meshes by local Galerkin projection. Computer Methods in Applied Mechanics and Engineering 200, 89-100.

Kaufmann, J., Bertram, V., 2011. Comparison of multi-reference frame and sliding interface propeller models for RANSE computations of shippropeller interaction. In: Proceedings of the 14th Numerical Towing Tank Symposium.

Kinnas, S. A., Chang, S.-H., He, L., Johannessen, J. T., 2009. Performance prediction of a cavitating rim driven tunnel thruster. In: First International Symposium on Marine Propulsors.

Kuiper, G., 1992. The Wageningen Propeller Series. MARIN. 
Lea, M., Thompson, D., Van Blarcom, B., Eaton, J., Friesch, J., Richards, J., 2003. Scale model testing of a commercial rim-driven propulsor pod. Journal of Ship Production 19 (2), 121-130.

Li, Y., Wang, F., 2007. Numerical investigation of performance of an axialflow pump with inducer. Journal of Hydrodynamics 19, 705-711.

Manna, M., Vacca, A., 2009. Torque reduction in Taylor-Couette flows subject to an axial pressure gradient. Journal of Fluid Mechanics 639, 373-401.

Petit, O., Page, M., Beaudoin, M., Nilsson, H., 2009. The ERCOFTAC centrifugal pump openfoam case-study. In: 3rd IAHR International Meeting of the Workgroup on Cavitation and Dynamic Problems in Hydraulic Machinery and Systems.

Sharkh, S. M., Turnock, S. R., Hughes, A. W., 2003a. Design and performance of an electric tip-driven thruster. In: Proceedings of the Institution of Mechanical Engineers, Part M: Journal of Engineering for the Maritime Environment. Vol. 217.

Sharkh, S. M., Turnock, S. R., Hughes, A. W., 2003b. Design and performance of an electric tip-driven thruster. Proceedings of the Institution of Mechanical Engineers, Part M: Journal of Engineering for the Maritime Environment 217, 133-147.

Yakovlev, A. Y., Sokolov, M. A., Marinich, N. V., 2011. Numerical design and experimental verification of a rim-driver thruster. In: Second International Symposium on Marine Propulsors.

Yamada, Y., 1962. Torque resistance of a flow between rotating co-axial cylinders having axial flow. Bulletin of JSME 5 (20), 634-642. 